\title{
Endogenous Endophthalmitis in a Two-Week-Old Neonate with Hypofibrinogenemia
}

\section{Anuradha Ganesh ${ }^{1 *}$, Ahlam Al-Hamhami' ${ }^{2}$, Maha Mameesh ${ }^{1,3}$, Sana Al-Zuhaibi ${ }^{1}$, Sameer Raniga $S^{4}$, Yasser Wali ${ }^{5,6}$ and Abdullah Al-Mujaini ${ }^{1}$}

${ }^{1}$ Departments of Ophthalmology, Sultan Qaboos University Hospital, Muscat, Sultanate of Oman

${ }^{2}$ Nizwa Hospital, Sultanate of Oman

${ }^{3}$ Faculty of Medicine, Alexandria University, Alexandria, Egypt

${ }^{4}$ Department of Radiology, Sultan Qaboos University Hospital, Muscat, Sultanate of Oman

${ }^{5}$ Department of Child Health, Sultan Qaboos University Hospital, Muscat, Sultanate of Oman

${ }^{6}$ Department of Pediatrics, Faculty of Medicine, Alexandria University, Alexandria, Egypt

*Corresponding Author: Anuradha Ganesh, Senior Consultant, Departments of Ophthalmology, Sultan Qaboos University Hospital, Muscat, Sultanate of Oman.
Received: August 14, 2020

Published: August 25, 2020

(C) All rights are reserved by Anuradha

Ganesh., et al.

\section{Abstract}

We report a two-week-old neonate with congenital hypofibrinogenemia and neonatal sepsis who developed left endogenous endophthalmitis. Gram positive bacillus Rothia dentocariosa and Gram negative bacillus E. coli were isolated from blood culture. Despite early initiation of both topical and systemic antibiotic therapy the baby had a deteriorating course and the eye was eviscerated. Keywords: Hypofibrinogenemia; Septicemia; Endogenous Endophthalmitis; R. dentocariosa; E. coli; Evisceration

\section{Introduction}

Sepsis continues to be an important cause of morbidity and mortality among neonates, particularly in very low birth weight infants [1]. Endogenous endophthalmitis is characterized by hematogenous dissemination of micro-organisms from a focus in the body, that in turn cross the blood-retina barrier and inoculate the intraocular tissues. Endophthalmitis in infants is rare (incidence of 4.42 cases per 100,000 live births) and unlike in adults is more likely to be endogenous [2]. The pathogens most commonly involved in neonatal endophthalmitis are Candida species, group B streptococci, P. aeruginosa and other Gram-negative bacteria [3].
Hereditary fibrinogen abnormalities may be Type I, afibrinogenemia or hypofibrinogenemia, which is characterized by absent or low plasma fibrinogen antigen levels, or Type II, dysfibrinogenemia which shows normal or reduced fibrinogen levels but is associated with reduced functional activity. While afibrinogenemia may be associated with a bleeding diathesis, patients with hypofibrinogenemia and dysfibrinogenemia are usually asymptomatic [4].

We describe a case of bacterial endogenous endophthalmitis in a two-week-old infant with congenital hypofibrinogenemia, who presented with recurrent bleeding from umbilical stump, and was 
admitted with a diagnosis of neonatal sepsis. He developed left endogenous endophthalmitis during hospitalization. Despite intensive topical and intravenous antibiotic therapy, the condition of the child worsened and the eye had to be eviscerated.

\section{Case Report}

A two-week-old child was brought by his parents to the emergency with continuous bleeding from the umbilical stump and irritability. On examination he was found to have elevated temperature at $39.9^{\circ} \mathrm{C}$ and was admitted for septic work up. The child was started on systemic intravenous antibiotics (cefotaxime $150 \mathrm{mg}$ q6h, cloxacillin $150 \mathrm{mg}$ q6h and gentamicin $6 \mathrm{mg}$ q8h). Laboratory investigations revealed highly deranged coagulation profile with prothrombin time above $300 \mathrm{sec}$ (normal range 10 - 12.6s) and activated partial thromboplastin time above $180 \mathrm{sec}$ (normal range 0.89 - $1.11 \mathrm{sec}$ ). Fibrinogen level was 0.5 IU (normal range 1.7 - 3.6 IU). Based on these findings he was diagnosed with congenital hypofibrinogenemia. Blood culture on admission grew gram negative bacilli $E$. coli. Intravenous vitamin $\mathrm{K} 2 \mathrm{mg}$ was added to the treatment regime, and packed red blood cells and cryoprecipitate were administered.

On the fifth day of hospitalization, the child was noted to have left eye redness, discharge and eyelid swelling, and was referred urgently to the pediatric ophthalmology service. Ophthalmic examination of the right eye (OD) was unremarkable. Examination OS was difficult due to severe lid edema. An Alfonso eyelid speculum was used to separate the eyelids and inspect the eyeball. Proptosis with limitation of eye movements in all direction, conjunctival hyperemia and chemosis, and hazy cornea due to edema was noted. A total hypopyon was noticed and no further details could be seen (Figure 1). B-scan ultrasound showed small-medium amplitude echoes throughout the vitreous. A diagnosis of endogenous endophthalmitis with panophthalmitis was made. Topical fortified gentamicin and cefuroxime q1h were commenced along with topical atropine $0.1 \% \mathrm{bid}$. Intravenous antibiotics were changed to meropenem $70 \mathrm{mg}$ q8h, vancomycin $50 \mathrm{mg}$ q8h, and clindamycin $20 \mathrm{mg} \mathrm{q} 6 \mathrm{~h}$. Magnetic resonance imaging (MRI) of the brain and orbit were performed and confirmed the clinical impression of endophthalmitis (Figure 2A and $2 \mathrm{~B}$ ). MRI brain revealed presence of subdural empyema (Figure $3 \mathrm{~A}$ and $3 \mathrm{~B}$ ). The child underwent a bifrontal craniotomy and evacuation of the subdural collection, analysis of which showed numerous WBC, 60\% lymphocytes and
40\% polymorphonuclear cells. Gram stain showed Gram negative bacilli $E$ coli. Repeat blood culture grew Gram positive bacillus Rothia dentocariosa.

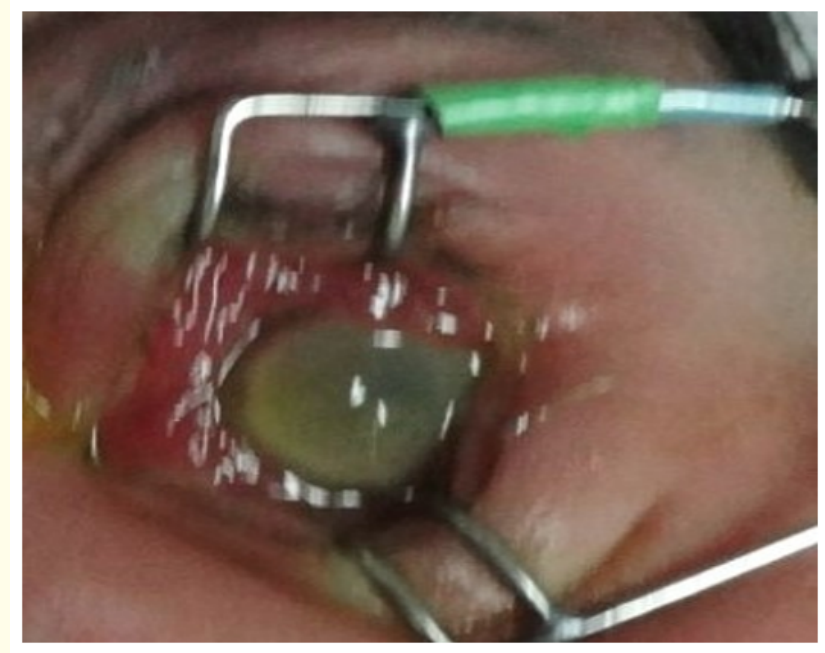

Figure 1: Anterior segment photo of left eye showing severe lid edema, conjunctival hyperemia and chemosis, hazy cornea and a total hypopyon.
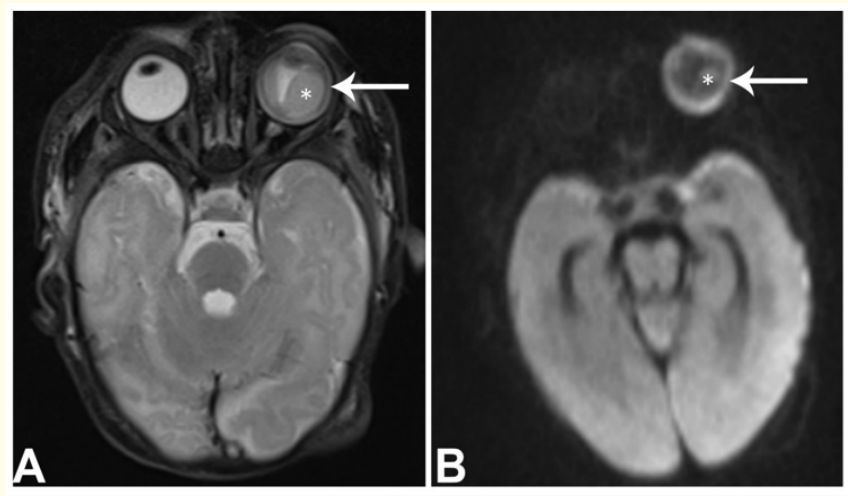

Figure 2: Axial A) fat-saturated T2W MRI of the orbit showing diffuse uveoscleral thickening of the left eye (arrow), retinal detachment and subretinal collection (asterix) suggestive of exudate and B) Diffusion-weighted MRI orbit showing an irregular and thick rim of diffusion restriction (arrow). 

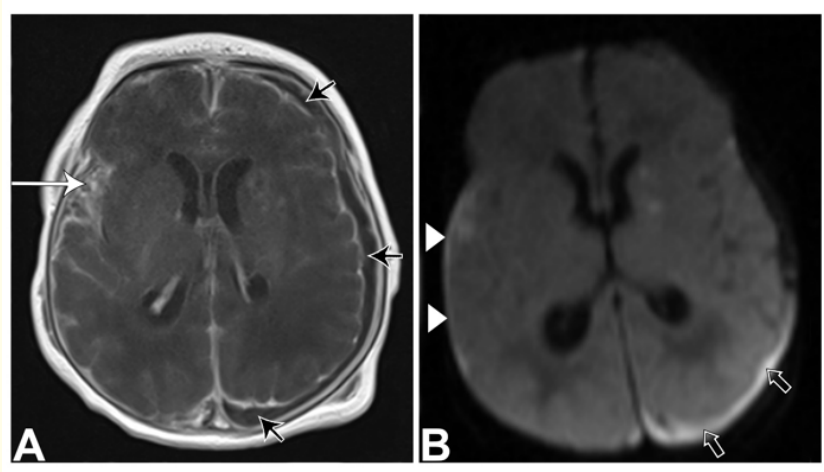

Figure 3: Axial A) post-contrast T1W MRI brain showing a subdural collection (black arrows) and diffuse leptomeningeal enhancement (white arrow) and B)

Diffusion-weighted MRI brain showing diffusion restriction (empty arrows, arrow-heads) suggestive of empyema.

On follow-up the ocular condition was seen to be improving with the child opening his eye spontaneously, improved ocular motility and reduced lid edema, conjunctival hyperemia and chemosis. On eight day of admission the cornea perforated spontaneously with partial extrusion of intraocular contents. Evisceration was performed and the child was fitted with a prosthetic eye.

\section{Discussion}

Endophthalmitis can be either endogenous or exogenous, depending on the route of infection. Exogenous endophthalmitis occurs when the eye infection develops as a result of corneal infection, perforating injury or intraocular surgery [5]. Endogenous endophthalmitis is relatively rare, accounting for only 2 - $8 \%$ of all endophthalmitis cases and results from hematogenous spread to the eye secondary to septicemia [5]. Endophthalmitis in infants is generally of an endogenous etiology $[2,7]$.

Neonatal endophthalmitis is a potentially blinding complication of neonatal sepsis [5]. Risk factors include systemic bacteremia, candidemia, low birth weight, respiratory infection, retinopathy of prematurity and blood transfusion [2]. Any disease process that results in septicemia has the potential to cause endophthalmitis. The bleeding umbilicus stump in our child is likely to have been the primary site of infection. The child showed signs of systemic sepsis before ocular manifestations of endophthalmitis, had no history of ocular trauma or surgery and had an intact ocular surface at initial presentation. These observations support a diagnosis of endogenous endophthalmitis. Although hypofibrinogenemia is not associated with spontaneous bleeding, the possibility of intraocular bleeding in an inflamed left eye due to hypofibrinogenemia with subsequent development of endophthalmitis cannot be excluded. Conjunctival swabs were negative; whereas blood cultures were positive for Gram positive bacillus $R$. dentocariosa and Gram negative bacillus E. coli.

Organisms previously implicated in endophthalmitis include P. aeruginosa, group B streptococci, $H$. influenzae type b, S. aureus, Salmonella enteritis, S. pneumoniae, N. meningitis and Candida species [5]. R. dentocariosa which was isolated from the blood culture in our patient is an aerobic gram-positive coccoid. It is a commensal of the oropharynx, and an unusual cause of infection in neonates [8]. There is only one report of ocular infection due to this organism in literature in a 11-year-old child who developed exogenous endophthalmitis following penetrating corneal injury [9].

The diagnosis of infectious endophthalmitis is based on the history and ophthalmic examination. Delayed referral is very common. A large series of neonatal endogenous endophthalmitis reported a mean interval of seven days between onset of symptoms and referral [10]. Early diagnosis of endophthalmitis in children can be challenging because they may not be able to verbalize symptoms such as decreased visual acuity, photophobia or pain. Systemic symptoms and signs which are more significant draw greater attention than ocular signs as was the case in our case. Often conjunctival hyperemia or discharge is mistaken for conjunctivitis and child is referred to the ophthalmologist only when manifestations worsen. Rarely endogenous endophthalmitis may be the first sign of systemic infection in an otherwise healthy patient [7]. In our patient with hypofibrinogenemia, the most plausible explanation of the rapid deterioration of his eye condition and lack of response to proper treatment is the presence of intraocular blood which acted as a culture medium for this virulent bacteria. Spontaneous bilateral subhyaloid hemorrhage in congenital hypofibrinogenemia has been previously reported [10].

In endogenous endophthalmitis $72 \%$ of patients will have a positive blood culture and is more likely to be a reliable indicator 
of causative organism [11,12]. A vitreous and aqueous tap may be considered in case of negative yield from blood culture [11]. The thick vitreous in children renders it difficult to aspirate with a needle, and may necessitate a pars plana vitrectomy to obtain a sufficient diagnostic sample of vitreous [13].

Therapeutic approach is determined by the extent of ocular involvement and the potential causative organism. The mainstay of treatment for endogenous endophthalmitis is culture report guided systemic antimicrobials at maximum recommended dose. Adjuvant intravitreal injection of antibiotics may be considered in severe cases unresponsive to systemic treatment $[3,11]$. The role of pars plana vitrectomy is controversial and is tailored to select patients [3]. Evisceration is indicated when the ocular condition is considered life-threatening. Primary implant is advocated as it spares the patient further surgery [14].

The visual prognosis of endogenous endophthalmitis is generally poor despite advances in therapy, with $30 \%$ of patients obtaining counting fingers or better final visual acuity and 16\% being enucleated [15]. Visual outcomes are generally more favorable among those surviving candidemia when compared with bacterial septicemia [3]. When the diagnosis is posterior diffuse metastatic endophthalmitis or panophthalmitis as in our case, blindness is the probable outcome regardless of treatment, and the patient or family should be made aware of this prognosis [12].

\section{Conclusion}

In summary, ocular symptoms and signs of endogenous endophthalmitis may be overshadowed by more prominent and lifethreatening manifestations of systemic infection in a neonate. A vigilant approach and a high degree of suspicion with early diagnosis and aggressive treatment is essential. Despite appropriate therapy, visual outcome in endogenous endophthalmitis is poor and may be associated with loss of the affected eye.

\section{Bibliography}

1. López Sastre JB., et al. "Neonatal invasive Candidiasis: A Prospective Multicenter Study of 118 Cases". The American Journal of Perinatology 20 (2003): 153-163.

2. Moshfeghi AA., et al. "Declining incidence of neonatal endophthalmitis in the United States". American Journal of Ophthalmology 151 (2011): 59-65.
3. Aziz HA., et al. "Intraocular infections in the neonatal intensive care unit case series". Clinical Ophthalmology 6 (2012): 733737.

4. De Moerloose P., et al. "Congenital fibrinogen disorders: an update". Seminars in Thrombosis and Hemostasis 39 (2013): 585-595.

5. Jackson TL., et al. "Endogenous Bacterial Endophthalmitis: A 17-year Prospective Series and Review of 267 Reported Cases". Survey of Ophthalmology 48 (2003): 403-423.

6. Basu S., et al. "Neonatal Endogenous Endophthalmitis: A Report of Six Cases". Pediatrics 131 (2013): 1292-1297.

7. Chaudhry IA., et al. "Pediatric endogenous bacterial endophthalmitis: case report and review of the literature". Journal of AAPOS 10 (2006): 491-493.

8. MacKinnon MM., et al. "A case of Rothia dentocariosa endophthalmitis". European Journal of Clinical Microbiology and Infectious Diseases 20 (2001): 756-757.

9. Partner AM., et al. "Rothia genus endophthalmitist following penetrating injury in a child". Eye 20 (2006): 502-503.

10. Pathengay A., et al. "Spontaneous bilateral subhyaloid hemorrhage in congenital hypofibrinogenemia". Retinal Cases and Brief Reports 5 (2011): 160-162.

11. Jalali S., et al. "Treatment outcomes and clinicomicrobiological characteristics of a protocol-based approach for neonatal endogenous endophthalmitis". European Journal of Ophthalmology 24 (2014): 424-436.

12. Greenwald MJ., et al. "Metastatic Bacterial Endophthalmitis: A Contemporary Reappraisal". Survey of Ophthalmology 31 (1986): 81-101.

13. Khan S., et al. "Pediatric infectious endophthalmitis: a review". The Journal of Pediatric Ophthalmology and Strabismus 51 (2014): 140-153.

14. Hui JI. "Outcomes of orbital implants after evisceration and enucleation in patient with endophthalmitis". Current Opinion in Ophthalmology 21 (2010): 375-379. 
15. Balaskas K and Potamitou D. "Endogenous endophthalmitis secondary to bacterial meningitis from Neisseria Meningitidis: a case report and review of the literature". Cases Journal 2

(2009): 149.

\section{Assets from publication with us}

- Prompt Acknowledgement after receiving the article

- Thorough Double blinded peer review

- Rapid Publication

- Issue of Publication Certificate

- High visibility of your Published work

Website: www.actascientific.com/

Submit Article: www.actascientific.com/submission.php

Email us: editor@actascientific.com

Contact us: +919182824667 